\title{
Globale Erdungssysteme: Bestimmung der Erdungsspannung (EPR) in Mittelspannungs-Kabelnetzen im einpoligen Fehlerfall
}

\author{
L. Fickert, T. Mallits, M. P. Resch OVE
}

\begin{abstract}
Die Umstellung der Erdschlussortungsmethode bzw. der Sternpunktsbehandlung führt in Mittelspannungs-Kabelnetzen zu steigenden Fehlerströmen und somit zu erhöhten Anforderungen an die Erdungssysteme hinsichtlich der Sicherstellung der Personensicherheit im Erdschluss-/Erdkurzschlussfall.

Basierend auf induktiv gekoppelten Leiterschleifen nach dem Modell von Carson und Pollaczek werden in einem ersten Schritt, auf vereinfachtem analytischen Weg, die Fehlerstromaufteilung und die Potentialverteilung bestimmt. In einem zweiten Schritt werden die Ergebnisse mit einer erweiterten unabhängigen Modellierung verglichen und vertiefte Analysen durchgeführt. Als Ergebnis werden die maßgebenden Einflussfaktoren auf die Strom- und Potentialverhältnisse in verbundenen (globalen) Erdungssystemen und deren praktische Bedeutung im einpoligen Fehlerfall abgeleitet.
\end{abstract}

Schlüsselwörter: Erdfehlerstrom-Reduktionsfaktor; globales Erdungssystem; Erdungsspannung; EPR; Erdkurzschluss; Erdschluss; Mittelspannungs-Kabelnetze; Erdfehlerstromaufteilung; Personensicherheit

\section{Global grounding systems: determination of the earth potential rise (EPR) in medium voltage cable networks in the event of a single pole fault.}

\begin{abstract}
The expansion of medium-voltage networks by cable lines, or changes in the earth fault location method or changes of the neutral point treatment increasingly lead to rising earth fault currents and earth potential rises (EPR) at the fault location.

Based on the inductively coupled conductor loops model according to Carson and Pollaczek, in a first step, the fault current distribution and the potential distribution are determined in a simplified analytical way. In a second step, the results are compared to an extended independent modelling and, with this, further in-depth analyses are performed. As a result, the decisive influencing factors for the current and potential conditions in connected (global) earthing systems and their practical significance in the event of a single-pole fault are derived.
\end{abstract}

Keywords: earth fault current reduction factor; global earthing system; earth potential rise (EPR); single phase faults; medium voltage cable grids; earth fault current distribution; safety of people

Eingegangen am 20. Dezember 2018, angenommen am 23. Jänner 2019, online publiziert am 6. Februar 2019 (c) The Author(s) 2019

\section{Einleitung}

In Mitteleuropa wird derzeit ein Großteil der MittelspannungsKabelnetze mit Erdschlusskompensation betrieben. Der Vorteil dieser Methode liegt im geringen Strom an der Fehlerstelle; nachteilig allerdings sind die aufwändige, für einen effizienten Netzbetrieb erforderliche Abschnittsortung (Tiefenortung) und die im Fehlerfall erhöhte Spannungsbeanspruchung der Betriebsmittel in den gesunden Außenleitern.

In der Mittelspannungs-Verteilnetzebene werden zunehmend Kabel verlegt, bei denen eine Lichtbogenlöschung aus physikalischen Gründen nicht möglich ist. Nach dem Isolationsdurchbruch kommt es in der Regel im Kabel zu einem stehenden Erdschluss, der bestehen bleibt, bis der betreffende Netzabschnitt geortet und abgeschaltet worden ist.

Beim Netzausbau mit Kabeln steigen nicht nur die kapazitiven Betriebsströme, sondern es rücken auch speziell die Erdschlussströme in den Interessenfokus. Es ist zu erwarten, dass in Zukunft Mittelspannungs-Verteilnetze (auch mit Freileitungsanteilen) mit ka- pazitiven Erdschlussströmen weit jenseits des bisher dafür üblichen

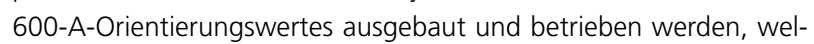
cher von vielen Netzbetreibern aus früheren Normen als vorgegebene Löschgrenze abgeleitet wurde. Da mit den steigenden kapazitiven Erdschlussströmen auch die Erdschluss-Restströme an der Fehlerstelle steigen und diese für die Potenzialanhebung der Fehlerstelle verantwortlich sind, ist die Bestimmung dieser Potenzialanhebung zur Aufrechterhaltung der Personensicherheit im Erdschlussfall erforderlich.

Zusätzlich zur Problematik der generell ansteigenden ErdschlussRestströme stellt sich hinsichtlich der Potenzialanhebung im Erd-

Fickert, Lothar, Technische Universität Graz, Institut für Elektrische Anlagen und Netze, Inffeldgasse 18/l, 8010 Graz, Österreich (E-Mail: lothar.fickert@tugraz.at); Mallits, Thomas, IES Institut für Elektrotechnik und Sicherheitswesen Ziviltechniker GmbH, Gastgebgasse 27, 1230 Wien, Österreich (E-Mail: mallits@ies-zt.at); Resch, Markus Patrick, Technische Universität Graz, Institut für Elektrische Anlagen und Netze, Inffeldgasse 18/I, 8010 Graz, Österreich (E-Mail: markus.resch@student.tugraz.at) 


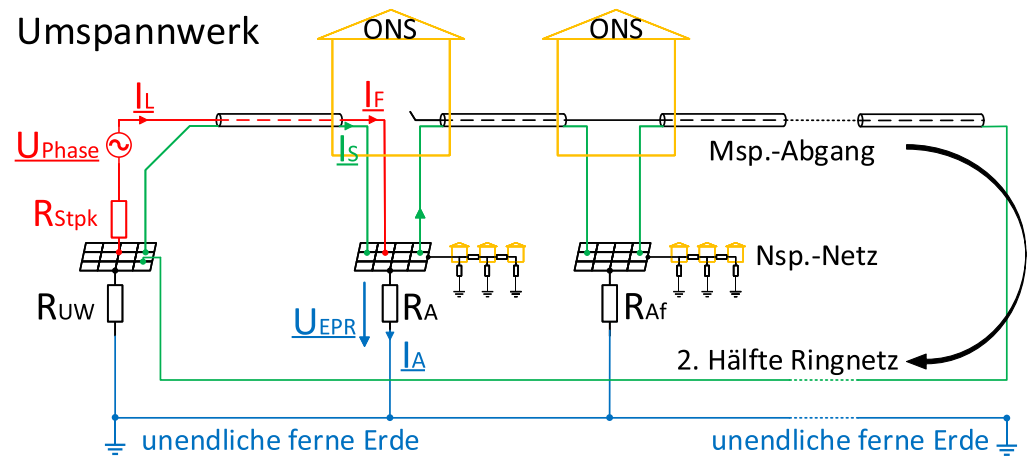

Abb. 1. Grundschaltung eines Netzabgangs in einem Ringnetz mit offener Trennstelle [4], rot - Fehlerschleife; grün - Kabelschirm; blau - Bezugserde; gelb - geerdete Bauwerke (Farbabbildung online)

schlussfall die Frage, inwieweit allfällige für die Fehlerortung zusätzlich eingespeiste Ströme die Personensicherheit negativ beeinflussen können. Diese Erhöhung des Erdfehlerstromes erfolgt z. B. beim Verfahren der Wattrestromerhöhung sowie der Kurzzeitigen Niederohmigen Sternpunktserdung - KNOSPE $[5,6]$. Speziell aber, wenn eine komplette Umstellung der Sternpunktserdung auf die Niederohmige Sternpunktserdung (NOSPE) mit Strömen in der GröBenordnung $300 \mathrm{~A}$ bis $1000 \mathrm{~A}$, fallweise bis $2000 \mathrm{~A}$ realisiert wird, ist die Frage nach der Potenzialanhebung im Erdfehlerfall relevant, da auf jeden Fall die notwendige Personensicherheit im einpoligen Erdschlussfall gewährleistet sein muss.

\section{Methodik der Bestimmung der Potenzialanhebung im Erdfehlerfall}

Schritt 1: Um in Mittelspannungs-Kabelnetzen die im einpoligen Fehlerfall auftretenden Ströme sowie die dadurch verursachten relevanten Erdungsspannungen (E्arth Potential Rise, EPR) zu bestimmen, wird auf der Basis der Grundgleichungen von J.R. Carson [1] und F. Pollaczek [2] in diesem Beitrag ein erweitertes Berechnungsmodell vorgestellt. Das Basismodell wird hierbei um die Ausbreitungswiderstände an der Fehlerstelle und die Einbeziehung weiterer, mit der Fehlerstelle leitend verbundener Erderstrukturen erweitert (siehe Abb. 1). Durch die Lösung des zugehörigen linearen Gleichungssystems erhält man im Sinne einer Worst-Case-Abschätzung eine Formel für den globalen bzw. lokalen Reduktionsfaktor. Damit werden die Erderströme und - da die Erdungsimpedanzen als bekannt vorausgesetzt werden - die resultierenden Erdungsspannungen bestimmt.

In diesem ersten Schritt wird die Fehlerschleife von der Quelle (Umspannwerk) bis zur Senke (Fehlerstelle) ohne Zwischenerdungen analysiert.

Schritt 2: Im zweiten Schritt wird exemplarisch der spannungsreduzierende Einfluss einer bzw. mehrerer Zwischenerdungen gezeigt. Abschließend wird eine Worst-Case-Berechnungsvorschrift abgeleitet, mit deren Hilfe sich die Potenzialanhebung an der Fehlerstelle ableiten lässt. Wenn diese Potenzialanhebung unter Einbeziehung der Abschaltzeiten des Schutzes die Berührungsspannungs-Kriterien gemäß der Europanorm EN 50522 [7] einhält, ist für den untersuchten Einzelfall ein globales Erdungssystem nachgewiesen.

\section{Modellierung des Netzes}

Als Ausgangspunkt für die Modellierung des Kabelnetzes wird angenommen, dass dieses als Ringnetz mit offener Trennstelle betriebenen wird. Es kann also ein fehlerhafter Abgang mit (mindestens)

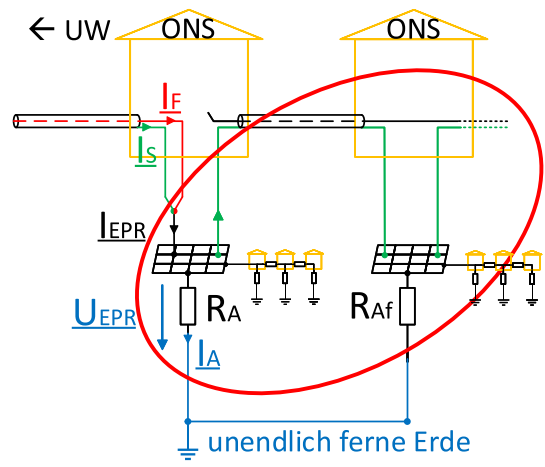

Abb. 2. Zusammenfassung der nachfolgenden Stationen und der Niederspannungsabgänge [4] (Farbabbildung online)

einem anderen Abgang zusammengeschaltet werden, der mit demselben Umspannwerk verbunden ist. Die durchverbundenen Schirme stellen also eine Ringstruktur dar (siehe Abb. 1).

Die Sternpunktsbehandlung ist im Folgenden ohne Beschränkung der Allgemeinheit als niederohmige Sternpunktserdung (NOSPE) ausgeführt.

Es wird ferner im Sinne der üblichen Netzpraxis vorausgesetzt, dass die Kabelschirme beidseitig direkt geerdet sind.

Zu beachten ist, dass der zu untersuchende Abschnitt in diesem ersten Schritt (siehe Kapitel 2. „Methodik”) ohne Zwischenerdungen (weitere geerdete Stationen) zwischen dem Umspannwerk und der Fehlerstelle modelliert ist und die Fehlerstelle somit in einer Ortsnetzstation am Ende des fehlerhaften Stiches angenommen wird. Voraussetzungsgemäß sind hier die beidseitig geerdeten Kabelschirme durchverbunden, sodass bezüglich der Erdungen stets ein geschlossener Ring vorliegt.

Alle mit der fehlerhaften Ortsnetzstation über die Kabelschirme verbundenen, "stromabwärts" gelegenen Stationen in der zweiten Hälfte des Ringes und die Niederspannungsabgänge der fehlerhaften Station werden gemäß Abb. 2 zusammengefasst. Dieser ErsatzAusbreitungswiderstand wird in der Folge mit $\underline{R}_{\mathrm{A}_{-}^{\prime}}$ bezeichnet.

Dieser Ersatz-Ausbreitungswiderstand ergibt sich aus der Parallelschaltung des eigentlichen Stations-Erdungswiderstandes $R_{\mathrm{A}}$ mit dem Kettenleiterwiderstand $\underline{R_{k e t t e}}$, der sich aus der Serienschaltung der nachfolgenden Kabelabschnitte (Längsimpedanzen) und der nachfolgenden Stationsausbreitungswiderstände $R_{\text {Af }}$ ergibt sowie dem Erdungswiderstand des von der fehlerhaften Station ver- 


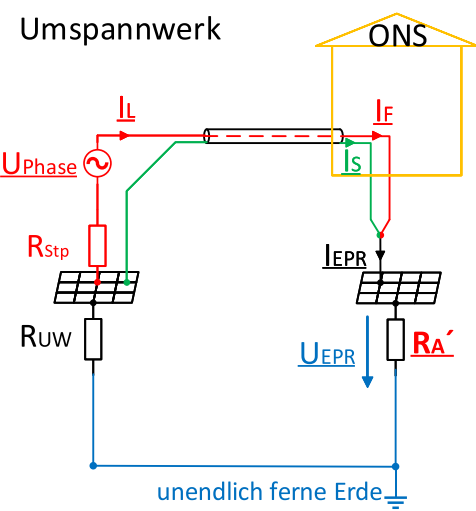

Abb. 3. Relevante Fehlerschleife [4] (Farbabbildung online)

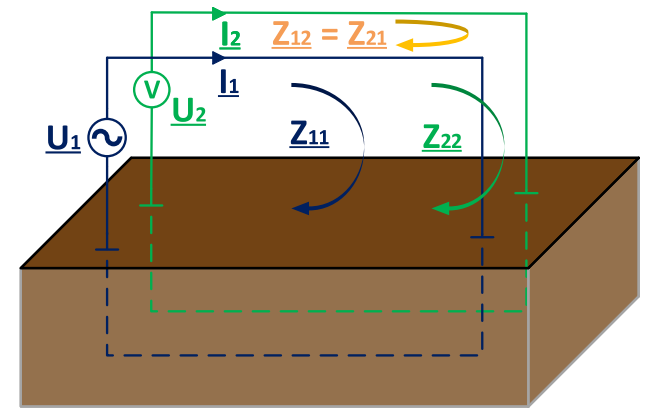

Abb. 4. Induktive Verkopplung zweier Schleifen mit Stromfluss über Erde [4] (Farbabbildung online)

sorgten Niederspannungsnetzes $R_{\mathrm{Nsp}}$

$$
\frac{1}{\underline{R_{A}^{\prime}}}=\frac{1}{R_{A}}+\frac{1}{\underline{R_{\text {Kette }}}}+\frac{1}{R_{\text {Nsp }}}
$$

Als Ergebnis erhält man die Ersatzschaltung gemäß Abb. 3, welche in der Folge mathematisch analysiert wird.

Das durch J.R. Carson und F. Pollaczek für die induktive Verkopplung zweier Schleifen mit Stromfluss über Erde beschriebene Gleichungssystem basiert auf der in Abb. 4 wiedergegebenen allgemeinen Grundschaltung. In Abb. 4 ist im konkreten Fall die Fehlerschleife die erste (aktive) Schleife (Index L) und die Schirm-Erde-Schleife die zweite (passive) Schleife (Index S).

Die dazugehörigen Grundgleichungen lauten:

$$
\begin{aligned}
& \underline{U_{1}}=\underline{Z_{11}} \cdot \underline{I_{1}}+\underline{Z_{12}} \cdot \underline{I_{2}} \\
& \underline{U_{2}}=\underline{Z_{21}} \cdot \underline{I_{1}}+\underline{Z_{22}} \cdot \underline{I_{2}}
\end{aligned}
$$

Die Spannungen $U_{1}$ und $U_{2}$ bezeichnen die an die jeweiligen Schleifen angelegten Spannungen. Während die aus der grundlegenden Theorie bekannten Impedanzen $\underline{Z}_{11}$ und $\underline{Z}_{22}$ die Selbstimpedanzen repräsentieren, stellen $\underline{Z}_{12}$ und $\underline{Z}_{21}$ die Koppelimpedanzen der beiden Schleifen, allerdings ohne Berücksichtigung der in der Realität vorhandenen Erdausbreitungswiderstände, dar.

\section{Anwendung auf die Netzpraxis}

Das in der Netzpraxis aus der Leiter-Erde-Schleife (Fehlerschleife) und der beidseitig geerdeten Kabelschirm-Erde-Schleife (Kurzschlussschleife) gebildete Leitungssystem bzw. die gegenseitige induktive Verkopplung beider Schleifen ist in Abb. 5 noch einmal in netztechnischer Form dargestellt.

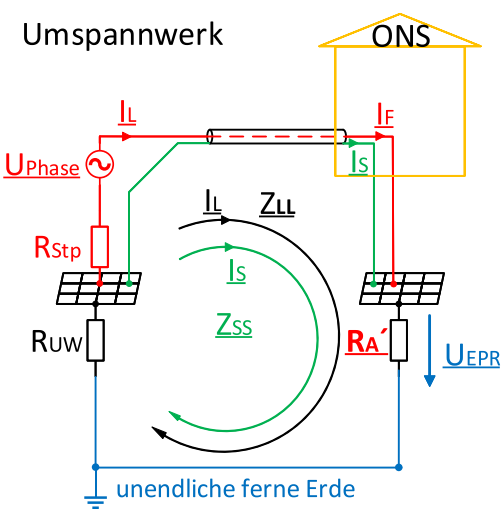

Abb. 5. Induktive Verkopplung der Fehlerschleife (rot) und der durch den beidseitig geerdeten Kabelschirm gebildeten Kurzschlussschleife (grün) [4] (Farbabbildung online)

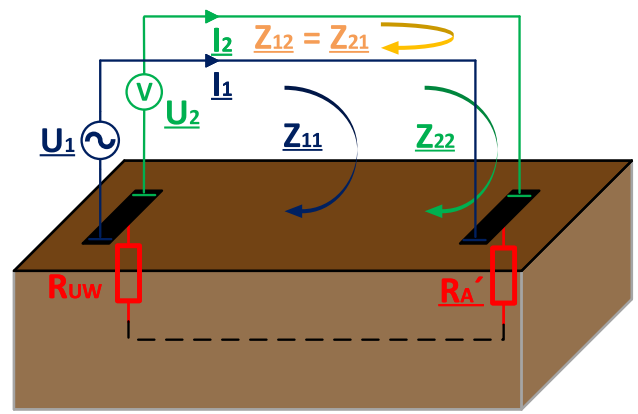

Abb. 6. Induktive Verkopplung zweier Leiter-Erde-Schleifen mit zusätzlichen Stationserdungswiderständen [4] (Farbabbildung online)

Die Grundschaltung aus Abb. 4 wird für die nachfolgenden Betrachtungen um die Ausbreitungswiderstände des Umspannwerks Ruw und des Ersatz-Ausbreitungswiderstand der Station $\underline{R}_{\mathrm{A}_{-}^{\prime}}$ erweitert (siehe Abb. 6).

\section{Rechenkern und Ergebnisse}

Das dazugehörige lineare Gleichungssystem ergibt sich durch Erweiterung der Gleichungen (2) und (3) und unter Berücksichtigung des beidseitig geerdeten Schirms, wodurch $\underline{U}_{2}=0$ gilt, wie folgt:

$$
\begin{aligned}
\underline{U_{\text {Phase }}}= & \left(\underline{z_{11}}+\underline{R_{\mathrm{A}}^{\prime}}+R_{\mathrm{uW}}+R_{\mathrm{Stpkt}}\right) \cdot \underline{L_{L}}+\left(\underline{z_{12}}+\underline{R_{\mathrm{A}}^{\prime}}+R_{\mathrm{UW}}\right) \cdot \underline{I_{\underline{S}}} \text { (4) } \\
& 0=\left(\underline{z_{21}}+\underline{R_{\mathrm{A}}^{\prime}}+R_{\mathrm{uW}}\right) \cdot \underline{L_{L}}+\left(\underline{z_{22}}+\underline{R_{\mathrm{A}}^{\prime}}+R_{\mathrm{uW}}\right) \cdot \underline{I_{S}}
\end{aligned}
$$

$\underline{\underline{L}}-$ - Strom des fehlerhaften Leiters (Erdfehlerstrom)

Is - Schirmstrom des fehlerhaften Kabels

$\bar{R}_{\text {Stp }}$ - Strombegrenzungswiderstand (NOSPE- Widerstand)

Ruw - Ausbreitungswiderstand des Umspannwerks

$\underline{U}_{\text {Phase }}$ - Leiter-Sternpunkt-Spannung (Phasenspannung des Transformators)

$\underline{Z}_{11}$ - Selbstimpedanz der fehlerhaften Leiter-Erde-Schleife (ohne Zusatzwiderstände)

$\underline{Z}_{12}=\underline{Z}_{21}$ - Koppelimpedanz zwischen der Leiter-Erde-Schleife und der Schirm-Erde-Schleife (ohne Zusatzwiderstände)

$\underline{Z}_{22}$ - Selbstimpedanz der Schirm-Erde-Schleife (ohne Zusatzwiderstände)

Der für die Potenzialanhebung wirksame (Gesamt-)Erdungsstrom IEPR, die Erdungsspannung $\underline{\text { UPR }}$ und der über die lokale Erdungsan- 
lage abfließende Erdungsstrom $\underline{I}_{\underline{A}}$ ergeben sich wie folgt:

$$
\begin{aligned}
& \underline{I_{\mathrm{EPR}}}=\underline{I_{L}}+\underline{I_{S}}=\underline{I_{\underline{L}}} \cdot \frac{\underline{Z_{S S}}-\underline{Z_{S L}}}{\underline{R_{A}^{\prime}}+\underline{Z_{S S}}+\underline{R_{U W}}} \\
& \underline{U_{E P R}}=\underline{I_{L}} \cdot \frac{R_{A}^{\prime} \cdot\left(\underline{Z_{S S}}-\underline{Z_{S L}}\right)}{\underline{R_{A}^{\prime}}+\underline{Z_{S S}}+\underline{R_{U W}}} \\
& \underline{I_{A}}=\underline{I_{L}} \cdot \frac{\underline{Z S S}_{R_{A}}^{\prime}+\underline{Z_{S S}}+\underline{R_{U W}}}{\underline{R_{U W}}} \cdot \frac{R_{A}^{\prime}}{\overline{R_{A}}}
\end{aligned}
$$

In diesem Zusammenhang ist es sinnvoll, zwei Reduktionsfaktoren zu definieren:

1.) Der für die Erdungsspannung relevante "globale" Reduktionsfaktor $\underline{r}_{\text {global }}$ beschreibt das Verhältnis zwischen dem Gesamterdungsstrom $\underline{I E P R}_{\text {R }}$ und dem Fehlerstrom $\underline{\underline{L}}$.

$$
\underline{r_{\text {global }}}=\frac{I_{\text {EPR }}}{\underline{I_{L}}}
$$

2.) Der "lokale" Reduktionsfaktor $\underline{r}_{\text {lokal }}$ hingegen ist durch das Verhältnis des in die lokale Erde abfließenden Erdungsstromes I. zum Fehlerstrom IL definiert.

$$
\underline{r_{\text {lokal }}}=\frac{\underline{I_{A}}}{\underline{I_{L}}}
$$

Die beiden Faktoren sind wegen der Parallelschaltung des unmittelbaren Stationserdungswiderstandes $R_{\mathrm{A}}$ mit den über die Kabelschirme verbundenen anderen Stationserdungswiderständen bzw. den mittels PEN-Leiter verbundenen Niederspannungserdungen immer unterschiedlich.

Der lokale Reduktionsfaktor ist grundsätzlich kleiner als der globale Reduktionsfaktor.

Wenn man bei der Detaillierung der Formel (6) die Terme zweiter Ordnung vernachlässigt, erhält man

$$
\underline{r_{\text {global }}}=\frac{l_{\text {EPR }}}{\underline{I_{\underline{L}}}}=\frac{\left[\left(r_{\mathrm{S}}^{\prime}\right)+j \cdot\left(x_{\mathrm{SS}}^{\prime}+x_{\mathrm{iS}}^{\prime}-x_{\mathrm{SL}}^{\prime}\right)\right] \cdot l}{\left[\left(r_{\mathrm{e}}^{\prime}+r_{\mathrm{S}}^{\prime}\right)+j \cdot\left(x_{\mathrm{SS}}^{\prime}+x_{\mathrm{iS}}^{\prime}\right)\right] \cdot 1+\underline{R_{\mathrm{A}}^{\prime}}+R \mathrm{UW}}
$$

I - Entfernung des Fehlerorts zum Umspannwerk

$R_{\mathrm{A}}^{\prime}$ - Ersatz-Ausbreitungswiderstand der Station (siehe GI. (1))

$\frac{R_{\mathrm{A}}^{\prime}}{r_{\mathrm{e}}^{\prime}}-$ spezifische Erdresistanz

$r_{\mathrm{S}}^{\prime}$ - spezifischer Widerstand des Schirmes bzw. (bei Parallelschaltung) der Schirme

$x_{\text {is }}^{\prime}$ - spezifische innere Selbstreaktanz der Schirm-Erde-Schleife (ohne Zusatzwiderstände)

$x_{S L}^{\prime}$ - spezifische Koppelreaktanz zwischen Leiter-Erde-Schleife und Schirm-Erde-Schleife

$x_{S S}^{\prime}$ - spezifische Selbstreaktanz der Schirm-Erde-Schleife (ohne Zusatzwiderstände)

Bezüglich der vollständigen Berechnungsvorschriften für $x_{i S}^{\prime}, x_{S L}^{\prime}$, $x_{S S}^{\prime}, r_{\mathrm{e}}^{\prime}$ und $r_{S}^{\prime}$ wird auf [4] verwiesen.

Unter Berücksichtigung realer Größenverhältnisse lässt sich dieser Ausdruck allerdings weiter vereinfachen zu:

$$
\underline{r_{\text {global }}}=\frac{l_{\text {EPR }}}{\underline{I_{L}}}=\frac{r_{\mathrm{S}}^{\prime} \cdot 1}{\left(r_{\mathrm{S}}^{\prime}+j \cdot x_{\mathrm{SS}}^{\prime}\right) \cdot 1+\underline{R_{\mathrm{A}}^{\prime}}+R_{\mathrm{UW}}}
$$

I - Entfernung vom Umspannwerk zur Fehlerstelle

$r_{S}^{\prime}$ - spezifischer Schirmwiderstand

$R_{\mathrm{A}}^{\prime}$ - Ersatzausbreitungswiderstand

$\overline{R_{u W}}$ - Ausbreitungswiderstand des Umspannwerks

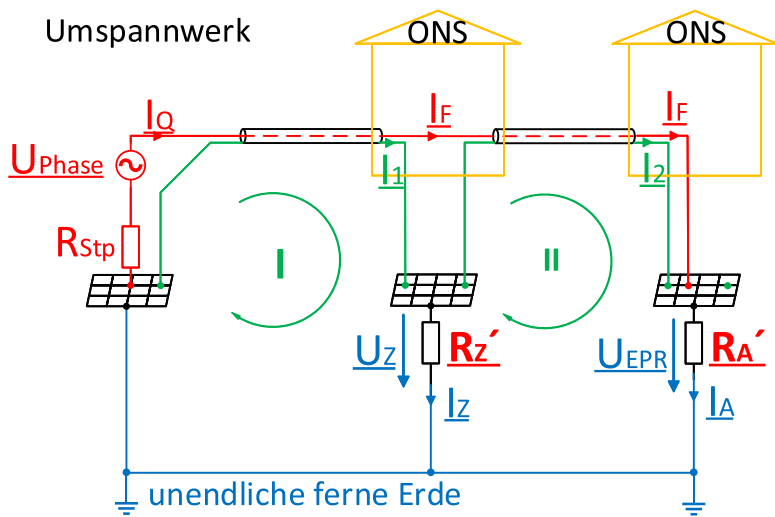

Abb. 7. Modell zur Berechnung des Einflusses einer einzigen Zwischenerdung [4] (Farbabbildung online)

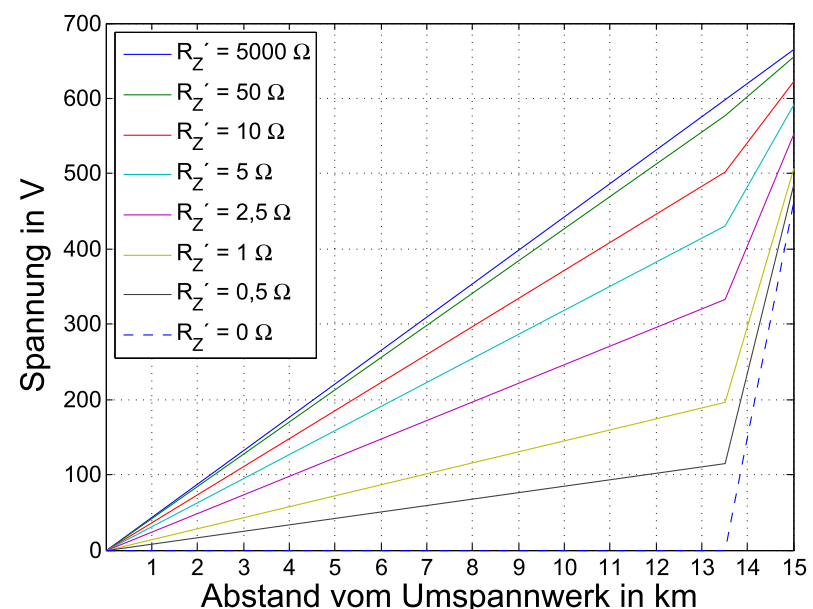

Abb. 8. Einfluss des Stationswiderstandes $\underline{R}_{-}^{\prime}$ auf die Stations-Erdungsspannung [4] (Farbabbildung online)

$$
\begin{aligned}
& x_{S S}^{\prime}-\text { spezifische Selbstinduktivität der Schirm-Erde-Schleife ge- }
\end{aligned}
$$

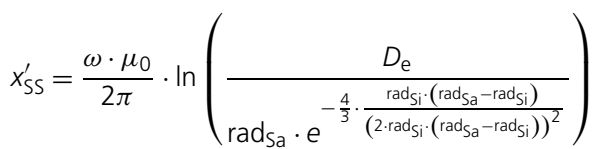

\section{Einfluss der Zwischenerdungen}

Um den Einfluss der im Allgemeinen zwischen dem Umspannwerk und der fehlerhaften Ortsnetzstation liegenden weiteren Ortsnetzstationen zu untersuchen, wird in einem ersten Schritt exemplarisch der Einfluss einer einzigen Ortsnetzstation untersucht (siehe Abb. 7).

Auch hier lassen sich gemäß [4] noch verhältnismäßig einfach analytische Gleichungen aufstellen. In der folgenden Abb. 8 ist der Einfluss einer zunehmend niederohmigen Zwischenerdung in einem Abstand von 10\% der Gesamtlänge I zur fehlerhaften ONS auf die Stations-Erdungsspannung dargestellt.

Man erkennt, dass mit sinkendem Ausbreitungswiderstand die Situation hinsichtlich Spannungsanhebung an der Fehlerstelle und 
auch der Spannungsverlauf entlang der fehlerhaften Leitung verbessert wird. Diese einfache Untersuchung zeigt, dass - was zu vermuten war - unmittelbar an der Fehlerstelle die größte Erdpotentialanhebung auftritt und dass jede zusätzliche Erdung als „EntlastungsErdung" die Spannungsanhebungen sowohl an der Fehlerstelle als auch entlang des Leitungszuges verringert. Somit stellt der in Abschnitt "5. Rechenkern und Ergebnisse" getätigte Ansatz eine leicht handhabbare Worst-Case-Betrachtung dar.

\section{Kabelnetze als globale Erdungssysteme}

Bereits in der vereinfachten Form (Gl. (12)) ist zu erkennen, dass der Ersatz-Ausbreitungswiderstand der Station $\underline{R}_{\mathrm{A}_{-}^{\prime}}$ sowie der Erdungswiderstand des Umspannwerks Ruw den globalen Reduktionsfaktor senken. Wichtig für die Analysen hinsichtlich der Existenz globaler Erdungssysteme ist aber vor allem die Tatsache, dass mit abnehmender Entfernung der Fehlerstelle zum Umspannwerk auch der globale Reduktionsfaktor praktisch linear abnimmt: Bei städtischen Kabelnetzen treten damit wegen der geringen Abstände zwischen einer fehlerhaften Ortsnetzstation und dem Umspannwerk sehr kleine Reduktionsfaktoren auf.

Diese Netze weisen unter Umständen auch bei Fehlerströmen in der Größenordnung von rund 1 kA in Verbindung mit den sehr niederohmigen Niederspannungsnetzen und unter Einbeziehung der Abschaltzeiten der Erdschluss-Schutzeinrichtungen in vielen Fällen die Eigenschaften eines globalen Erdungssystems auf [7]. Der entsprechende Nachweis ist aber im Einzelfall stets durch exemplarische Versuche im Verbindung mit den oben beschriebenen Berechnungen zu führen.

Da in städtischen Netzen insgesamt die Erdungsspannungen aus den obigen Gründen üblicherweise sehr klein sind und die abgreifbaren Berührungs- und Schrittspannungen wiederum nur Teile der Erdungsspannung betragen, kann bei Vorliegen entsprechend kleiner Reduktionsfaktoren in solchen Netzen vom Vorhandensein eines globalen Erdungssystems ausgegangen werden.

\section{Anwendungsbeispiel 1: Urbanes Mittelspannungs-Kabelnetz}

Das folgende Beispiel mit Zahlenwerten, welche exemplarisch für ein großstädtisches 20-kV-Netz mit der Sternpunktsbehandlung NOSPE und einem mittleren Stationsabstand von $300 \mathrm{~m}$ stehen, möge die im Rahmen dieser Arbeit gezeigte Vorgangsweise erläutern:

Eingangsparameter:

- $R_{\mathrm{A}}=1 \Omega$

- $R_{\mathrm{Nsp}}=0,2 \Omega$

- $R_{\text {Stpkt }}=10 \Omega$

- $\operatorname{Ruw}=0,1 \Omega$

- I = 1800 m (ohne Berücksichtigung der Zwischenerdungen, Worst-Case)

- Kabel (Aluminium-Leiter mit Kupferschirm) E-A2XHC2Y 150/RM25

Es ergeben sich im einpoligen Fehlerfall folgende Ströme und Spannungen:

- $\mathrm{L}=1070 \mathrm{~A}$

- $I_{\mathrm{S}}=930 \mathrm{~A}$

- $l_{\text {EPR }}=350 \mathrm{~A} \rightarrow r_{\text {global }}=0,33$

- $I_{\mathrm{A}}=32 \mathrm{~A} \rightarrow r_{\text {lokal }}=0,03$

- $U_{\text {EPR }}=32 \mathrm{~V}$

Die Potenzialanhebung der fehlerhaften Station beträgt somit nur $32 \mathrm{~V}$ und liegt damit weit unter dem Grenzwert von $80 \mathrm{~V}$, der in Europanorm EN 50522 [7] für die Berührungsspannung angegeben wird, wenn die Stromflussdauer beträchtlich länger als $10 \mathrm{~s}$ ist.
Die Berührungsspannungen in unmittelbarer Umgebung der Station und auch die über den PEN-Leiter ins Niederspannungsnetz transferierte Spannungsanhebung sind durch die im Zuge der betrachteten Strompfade relevanten Widerstände stets kleiner als die Spannung $U_{\text {EPR. }}$. Somit ist ein solches Netz als globales Erdungssystem zu klassieren.

\section{Anwendungsbeispiel 2: Rurales Mittelspannungs-Kabelnetz}

In diesem Beispiel werden exemplarisch Zahlenwerte für ein ländliches 20-kV-Kabelnetz mit der Sternpunktsbehandlung NOSPE und einem mittleren Stationsabstand von 2000 m angenommen.

Eingangsparameter:

- $R_{\mathrm{A}}=1 \Omega$

- $R_{\mathrm{Nsp}}=1 \Omega$

- $R_{\text {Stpkt }}=10 \Omega$

- $R_{\mathrm{UW}}=0,1 \Omega$

- I= 30 km (ohne Berücksichtigung der Zwischenerdungen, Worst(ase)

- Kabel (Aluminium-Leiter mit Kupferschirm) E-A2XHC2Y 150/RM25

Es ergeben sich im einpoligen Fehlerfall folgende Ströme und Spannungen:

- $\mathrm{L}_{\mathrm{L}}=500 \mathrm{~A}$

- $I_{S}=450 \mathrm{~A}$

- $l_{\text {EPR }}=170 \mathrm{~A} \rightarrow r_{\text {global }}=0,35$

- $I_{\mathrm{A}}=61 \mathrm{~A} \rightarrow r_{\text {lokal }}=0,12$

- $U_{\mathrm{EPR}}=61 \mathrm{~V}$

Hier beträgt die Potenzialanhebung der fehlerhaften Station $61 \mathrm{~V}$, und liegt somit über dem Wert aus dem Beispiel in Punkt 8. Dies lässt sich durch die Annahme eines größeren Widerstandswertes für die mit den Ortsnetzstationen verbundenen Niederspannungsnetze erklären. Allerdings liegt auch die Potentialanhebung von $61 \mathrm{~V}$ wieder unter dem oben angeführten Grenzwert für die Berührungsspannung laut Europanorm EN 50522 [7].

Im Betrieb eines Netzes wird die Personensicherheit gemäß Europanorm EN 50522 [7] zusätzlich erhöht, wenn die Einwirkungszeit in einem allfälligen Berührfalle durch eine entsprechende selektive Schutzabschaltung herabgesetzt wird. Im praktischen Netzbetrieb sind Zeiten von maximal 1 Sekunde erzielbar.

\section{Erweiterte Modellierung}

Im Rahmen einer erweiterten Modellierung wurde ein modulares Phasengrößenmodell mit erhöhtem Detailgrad realisiert [3]. Das Modell ermöglicht durch modulare Blöcke (Umspannwerk mit Spannungsquellen, Kabelstrecken mit beidseitig geerdeten Schirmen und einem erdfühligen Begleiterder, ONS mit der Fehlerstelle) einen dreiphasigen Aufbau nahezu beliebiger MittelspannungsKabelnetzstrukturen (Stich-, Ring- und Maschenstrukturen) und dessen erdungswirksamer Komponenten (z. B. Begleiterder). Die Besonderheit des in [3] beschriebenen Ansatzes liegt in der zweckmäßigen Modellierung der induktiven Verkopplungen der Leiter, Schirme und des Begleiterders (siehe Abb. 9).

Die induktive Verkopplung der erweiterten Modellierung (siehe orange Pfeile in Abb. 9) wird mittels der Kreuzschaltung nach E. Clarke [8] realisiert (siehe Abb. 10), welche in Folge um die wirksamen Kabelkapazitäten erweitert wird.

Im Rahmen der Analysen in [3] werden folgende Parameter

- Mittelspannungsnetz-Struktur

- Niederspannungsnetz Struktur 


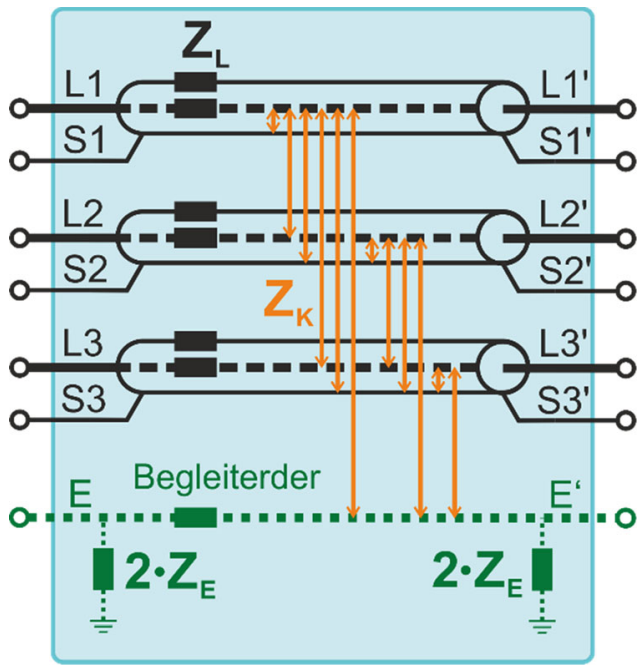

Abb. 9. Modellierung der Kabelstrecke mit Begleiterder $Z_{K}-$ Koppelimpedanzen $Z_{\mathrm{E}}$ - Erdungsimpedanz des Begleiterders $Z_{\mathrm{L}}$ - Längsimpedanzen (Farbabbildung online)
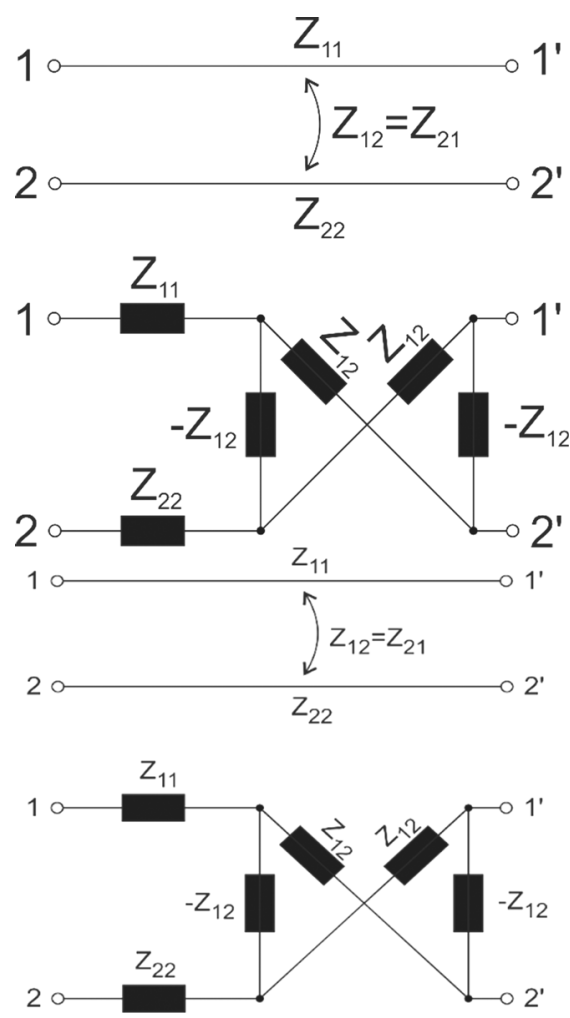

Abb. 10. Kreuzschaltung nach Clarke [3]

- Anzahl der Ortsnetzstationen

- Abstand der Ortsnetzstationen

- Ausbreitungswiderstand der ONS

- Lage des Fehlerorts

- Kabelparameter

- Verlegeart

- Begleiterder-Strukturen

durch eine große Anzahl von Variantenrechnungen vertiefend auf ihre Verallgemeinerung untersucht und dargestellt.

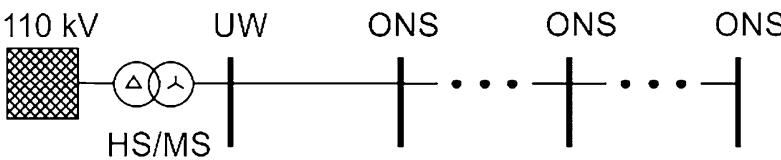

Abb. 11. Leitungsstich mit einem einpoligen Fehler am Ende [3]

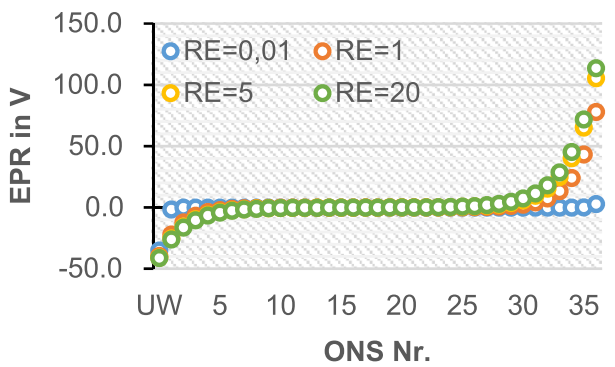

Abb. 12. Erdungsspannungen in Abhängigkeit der Ausbreitungs-widerstände (Farbabbildung online)

Abbildung 11 zeigt beispielhaft eine Mittelspannungs-Kabelstichstruktur, anhand welcher der Einfluss der ONS-Ausbreitungswiderstände (symmetrische Variation) auf die Erdungsspannungen analysiert wird.

Das Berechnungsergebnis in Abb. 12 verdeutlicht, dass Ausbreitungswiderstände größer als ca. $10 \Omega$ die Potentialverhältnisse im System nur mehr gering beeinflussen.

Hinsichtlich der vereinfachten Modellierung gemäß Kapitel 3 bestätigen die umfangreichen Untersuchungen in [3], dass eine einphasige Modellierung des Kabelsystems (bei Anpassung des wirksamen Schirmquerschnittes) mit guter Näherung zulässig ist.

Weiters wird in [3] gezeigt, dass die vereinfachte Modellierung gemäß Kapitel 3 zu einer Überschätzung der Potentialanhebung führt und somit hinsichtlich Berührungsspannungen auf der sicheren Seite liegt.

\section{Zusammenfassung}

Der Ausbau von Mittelspannungsnetzen mit Kabeln kann bereits ohne, insbesondere aber bei Umstellung der Sternpunktsbehandlung zu steigenden einpoligen Fehlerströmen führen, welche ihrerseits steigende Erdungsspannungen in der unmittelbaren und - durch Verschleppung - mittelbaren Umgebung der Fehlerstelle hervorrufen.

Zur grundlegenden Berechnung der Strom- und Potentialverhältnisse im Erdkurzschluss- bzw. Erdschlussfall werden Analysen mittels eines vereinfachten analytischen Ansatzes durchgeführt. Mit Hilfe eines erweiterten modularen Phasengrößenmodells werden die Grundlagen des analytischen Ansatzes validiert und erweiterte Detailanalysen durchgeführt.

Als Ergebnis erhält man einerseits analytische Formeln zur Bestimmung der Erdungsspannungen, welche sich zur Worst-CaseAbschätzung eignen, und andererseits ein MATLAB-basiertes modulares Phasengrößenmodell, mittels dem die maßgeblichen Einflussfaktoren aufgezeigt werden können.

\section{Danksagung}

Open access funding provided by Graz University of Technology.

Open Access This article is distributed under the terms of the Creative Commons Attribution 4.0 International License (http://creativecommons.org/ 
licenses/by/4.0/), which permits unrestricted use, distribution, and reproduction in any medium, provided you give appropriate credit to the original author(s) and the source, provide a link to the Creative Commons license, and indicate if changes were made.

\section{Literatur}

1. Carson, J. R. (1926): Wave propagation in overhead wires with ground return. Bell Syst Techn. J., 5, 539-554.

2. Pollaczek, F. (1926): Über das Feld einer unendlich langen wechselstromdurchflossenen Einfachleitung. Elekt. Nachrichtentech., 9, 339-359.

3. Mallits, T. (2018): Fehlerstromaufteilung und Potentialverhältnisse in komplexen (Globalen-) Erdungssystemen und deren Einfluss auf die Beurteilung. Dissertation, Institut für Elektrische Anlagen und Netze, TU Graz.

\section{Autoren}

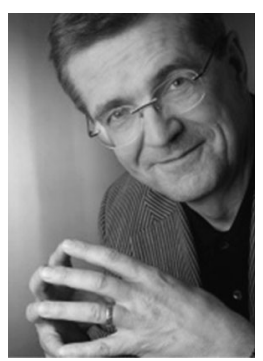

\section{Lothar Fickert}

Jahrgang 1949, Studium der Elektrotechnik an der Technischen Universität Wien, Österreich; Tätigkeit in verschiedenen Positionen bei internationalen und österreichischen Unternehmen (Elin Union, Brown Boveri, WIENSTROM), seit 1998 an der Technischen Universität Graz, 1999-2017 Vorstand des Institutes für Elektrische Anlagen und Netze. Mitarbeit: OVE, CENELEC, CIGRE.

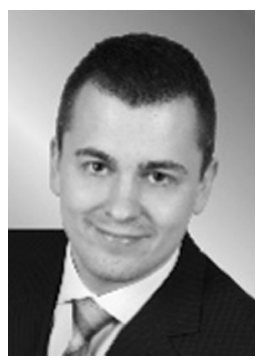

\section{Thomas Mallits}

wurde 1984 in Bruck/Mur, Österreich, geboren; Studium der Elektrotechnik an der Technischen Universität Graz, Österreich, Diplomarbeit am Institut für Elektrische Anlagen. Thomas Mallits arbeitete von 2011 bis 2018 am Institut für Elektrische Anlagen der Technischen Universität Graz und ist seither am IES Institut für Elektrotechnik und Sicherheitswesen Ziviltechniker $\mathrm{GmbH}$ beschäftigt.
4. Resch, M. P. (2018): Berechnung und Messung von Erdfehlerstrom-Reduktionsfaktoren bei einpoligen Erdfehlern. Institut für Elektrische Anlagen und Netze, TU Graz. Masterarbeit.

5. Mallits, Th., Fickert, L., Schmautzer, E. (2017): Globales Erdungssystem in urbanen Kabelnetzen. E\&I 8, 2017

6. Fickert, L., Steurer, H., Pasker, J., Raunig, C., Mallits, T. (2014): Stromstarke präzise (Abschnitts-) Ortung im Erdschlussfall. ETG-Fachbericht 143: STE 2014, Sternpunktbehandlung in Netzen bis $110 \mathrm{kV}$ (D-A-CH). VDE Verlag: Berlin.

7. ÖVE/ÖNORM EN 50522: Erdung von Starkstromanlagen mit Nennwechselspannungen über 1 kV. Ausgabe: 2011-12-0

8. Clarke, E. (1950): Circuit analysis of A-C power systems. Symmetrical and related components (Vol. I, S. 37). New York: Wiley.

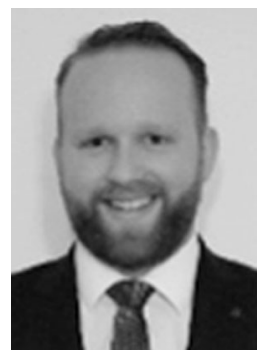

\section{Markus Patrick Resch}

wurde 1994 in Graz geboren. Studium der Elektrotechnik an der Technischen Universität Graz, Österreich. Diplomarbeit im Bereich Schutztechnik am Institut für Elektrische Anlagen und Netze der TU Graz. 